\title{
¿Qué es Trabajo Social? Los primeros vínculos de los estudiantes con la profesión: experiencias de Argentina, Brasil, Paraguay y Uruguay
}

\author{
Silvia Orieta Rivero-Rodríguez \\ Doctora en Ciencias Sociales. Licenciada en Trabajo Social \\ Universidad de la República. Montevideo, Uruguay \\ https:/ / orcid.org/0000-0003-1062-4899 • silviariv@gmail.com
}

\section{Leonel Del Prado}

Doctor en Ciencias Sociales. Licenciado en Trabajo Social

Universidad de la República. Paysandú, Uruguay

https:/ / orcid.org/0000-0003-1330-6642・leonelts@hotmail.com

\author{
Nidia Graciela Battilana-Amarilla \\ Magíster en Trabajo Social. Licenciada en Trabajo Social \\ Universidad Nacional de Asunción. San Lorenzo, Paraguay \\ https://orcid.org/0000-0002-2013-1468・ni.battilana@gmail.com
}

\section{Rosilaine Coradini-Guilherme}

Doctora en Servicio Social. Asistente Social

Universidade Federal do Pampa. São Borja, Brasil

https:/ / orcid.org/0000-0001-8705-8667 • rosilaineguilherme@yahoo.com.br

\section{Resumen}

Este artículo tiene como objetivo reflexionar sobre los primeros contactos que poseen las/os estudiantes con el Trabajo Social en su proceso de formación. Para ello se toman como insumo de análisis los programas de las materias introductorias de cuatro países latinoamericanos (Argentina, Brasil, Paraguay y Uruguay). El trabajo muestra que a pesar de las diferencias de cada país, dicha formación posee énfasis en posiciones que se enmarcan en la corriente cientificista y crítica.

Palabras clave: Trabajo Social; Formación Profesional; Trabajo Social Latinoamericano; Trabajo Social Contemporáneo.

Recibido: 22/03/2021 | Aprobado: 26/09/2021 | Publicado: 01/01/2022

cc) (i) Esta obra está bajo una Licencia Creative Commons Atribución-NoComercial-CompartirIgual 4.0 Internacional.

Financiación o proveniencia del artículo: Artículo vinculado al proyecto de investigación en curso La formación en Trabajo Social desde una perspectiva Latinoamericana. Financiado y avalado por la Universidad de la República (Uruguay), la Universidad Nacional de Asunción (Paraguay) y la Universidade Federal do Pampa (Brasil).

¿Cómo citar este artículo? / How to quote this article?

Rivero-Rodríguez, S. O., Del Prado, L., Battilana-Amarilla, N. G., y Coradini-Guilherme, R. (2022). ¿Qué es Trabajo Social? Los primeros vínculos de los estudiantes con la profesión: experiencias de Argentina, Brasil, Paraguay y Uruguay. Prospectiva. Revista de Trabajo Social e intervención social, (33), 211-234. doi: 10.25100/prts.v0i33.11092. 


\title{
What is Social Work? Students' First Approach with the Profession: Experiences from Argentina, Brazil, Paraguay and Uruguay
}

\begin{abstract}
This paper aims to reflect on the first contact that students have with Social Work in their educational process. The introductory course programs of four Latin American countries (Argentina, Brazil, Paraguay and Uruguay) are used as input for analysis. The work shows that despite the differences in each country, the educational training has an emphasis on positions that are framed in the scientific and critical school of thought.

Keywords: Social Work; Professional Training; Latin American Social Work; Contemporary Social Work.

Sumario: 1. Introducción, 2. Metodología, 3. Hallazgos, 4. Conclusiones, 5. Referencias bibliográficas.
\end{abstract}




\section{Introducción}

El objetivo que perseguimos en el presente trabajo es detallar y analizar con qué contenidos y con qué autores se vinculan los estudiantes de las licenciaturas ${ }^{1}$ de Trabajo Social $^{2}$ en sus primeros contactos con la profesión; para ello se consideran las materias introductorias de diferentes países: Argentina, Brasil, Paraguay y Uruguay ${ }^{3}$. Con el objetivo de tener una visión regional del tema se toman para el análisis la Universidad Nacional de Entre Ríos (Argentina), la Pontifícia Universidade Católica do Rio Grande do Sul (Brasil), Universidade Federal do Pampa (Brasil), la Universidad Nacional de Asunción (Paraguay) y la Universidad de la República (Uruguay) ${ }^{4}$, y el análisis de cada facultad es realizado por investigadores de cada uno de los países involucrados.

Este trabajo es parte de un proyecto de investigación sobre la formación en Trabajo Social e incorpora un conjunto de reflexiones que nos implican como docentes de equipos de cátedras de "Introducción al Trabajo Social" / "Fundamentos de Trabajo Social" de las carreras de Trabajo Social de Brasil, Paraguay y Uruguay. Éstas refieren al análisis de los autores que poseen mayor influencia en la formación actual de las licenciaturas de Trabajo Social en las Facultades analizadas. Para ello y con el objetivo de aportar a la construcción de un canon de autores reconocidos y conocidos por la comunidad profesional, analizamos los programas de las asignaturas específicas de Trabajo Social, ya que como se señala en Rivero y Del Prado (2018), para pensar los autores y temas clásicos es necesario centrarse en qué es lo que se enseña en las instituciones de formación universitaria, sin perjuicio de que se puedan tener otras perspectivas, entendemos, tomando la noción de "paradigma" de Thomas Kuhn (2006) y "campo científico" de Pierre Bourdieu (2008), que es importante hacer énfasis en el proceso de formación de los estudiantes, futuros profesionales, así como en el proceso en el que los estudiantes internalizan el paradigma o hacen habitus el campo profesional.

El artículo se organiza del siguiente modo: a) analizamos cada país en orden alfabético, detallando formación en el país, espacio de la materia en el contexto del programa y temas trabajados; b) reflexión en torno a potencialidades, fortalezas y vacíos que se encuentran en estos primeros momentos de formación de profesionales en Trabajo Social.

1 Es importante observar la correcta traducción del término licenciatura (diploma de bacharel) para no confundir, en caso brasilero, con las carreras de licenciaturas pedagógicas del área de educación.

2 Entre los países latinoamericanos encontramos la carrera profesional con el nombre de Trabajo Social, pero en Brasil se identifica con la nomenclatura Serviço Social.

3 "Introducción al Trabajo Social", "Fundamentos de Trabajo Social” y / o "Trabajo Social 1". De dichas materias tomamos los textos que aparecen referenciados como bibliografía obligatoria, la que frecuentemente se solicita que estudien los estudiantes; dejando de lado la bibliografía optativa, de consulta o de profundización.

4 Reflexiones actuales y más amplias sobre la profesión en el contexto latinoamericano se pueden encontrar en Melano y Deslauriers (2012). 
Rivero-Rodríguez, Del Prado, Battilana-Amarilla y Coradini-Guilherme

\section{Metodología}

Este artículo es un primer producto del estudio sobre la influencia del Movimiento de Reconceptualización en la formación de grado en Trabajo Social. El mismo surge de la iniciativa de los docentes del DTS, FCS, UDELAR (uno de ellos egresado de la UNER) y se integran a la propuesta docentes de la UNA y de UNIPAPA. Se pretende realizar una comparación de los formatos curriculares de esas unidades académicas para analizar el impacto de este Movimiento en las propuestas formativas.

El estudio pretende establecer un espacio de debate, en principio en esas unidades académicas, que nos permita entender las similitudes y diferencias de los diferentes procesos. El siguiente análisis se centrará en las formas en que se desarrollan las prácticas preprofesionales.

\section{Hallazgos}

Entendemos que aún no estamos en condiciones de presentar resultados finales, sin embargo, podemos aventurar algunas consideraciones fruto de estas primeras instancias de análisis. Sabemos que nuestro análisis se circunscribe solamente a las unidades académicas analizadas y por tanto, no tiene pretensiones de exhaustividad.

En principio, entendemos que el Movimiento de Reconceptualización ha sido fundamental en la reformulación del contenido de la formación de la disciplina. Los diferentes momentos en que se han incorporado estos cambios parecería estas asociados a los procesos de formación de posgrado de los docentes de las Unidades Académicas. Por tanto, en principio, podemos pensar que es posible que el Movimiento de reconceptualización instalara el proceso de reflexión sobre los contenidos de la disciplina y que la formación de posgrado de los docentes facilitaría la forma en que estos se traducen en la formación de grado.

\section{¿Qué se enseña?}

En este espacio se analiza la formación brindada por cada una de las unidades académicas analizadas, se coloca cada país en orden alfabético. Se detallan los objetivos respecto a cada oferta de formación, el lugar de la asignatura en la currícula, características del contenido y análisis de la bibliografía obligatoria.

\section{Argentina}

El Trabajo Social surge en Argentina en la década del '20 del siglo pasado. Estela Grassi (1989, p. 182), en referencia a los inicios de la profesión en Argentina, establece la 
Rivero-Rodríguez, Del Prado, Battilana-Amarilla y Coradini-Guilherme

existencia de lo que denomina como "dos líneas paralelas": una, que la definía como carrera para-médica, y, otra, como para-jurídica. Según la autora, “ambas líneas tuvieron su correlato institucional en la Universidad de Buenos Aires: la primera, en lo que fue el curso de Visitadoras de Higiene, luego la Licenciatura en Servicio Social en Salud, en la Facultad de Ciencias Médicas, y, la segunda, en la Escuela de Asistentes Sociales de la Facultad de Derecho, hasta 1985, en que la carrera pasó a depender del Rectorado, para ser integrada en una proyectada Facultad de Ciencias Sociales" (Grassi, 1989, p. 182).

En la actualidad, en Argentina se cuentan alrededor de 50 carreras de Trabajo Social, siendo la heterogeneidad y diversidad una de sus características principales (Alayón, 2007; Garma, Acevedo y Peralta, 2013; Rozas-Pagaza, 2017), más allá de distintos esfuerzos institucionales de la Federación Argentina de Unidades Académicas de Trabajo Social (FAUATS) en constituir una estructura curricular en todo el país, proceso que ha sido logrado con distintos grados de avances. Rozas-Pagaza (2017, p. 94) refiere: “En el país conviven más de 20 carreras en el ámbito de la Universidad -público / nacionales la mayoría- y privadas; y más de 30 en el ámbito de la Educación Superior No Universitaria o Terciaria, -pública- provinciales y privadas (dependientes de Ministerios provinciales de Salud, Acción Social y Educación o de instituciones confesionales). Muchas de ellas aisladas de los debates actuales y alejadas de la Organización Nacional". Alayón (2007, p. 20) lo expresa de la siguiente manera:

...el Trabajo Social que hoy conocemos está conformado por esta diversidad de orientaciones, que parecerían en algunos casos, decididamente irreconciliables, derivadas de la oscilación entre dos extremos de la función social de su práctica: la legitimación o el cuestionamiento del orden social vigente en un período histórico determinado.

En la actualidad, con la implementación de la Ley Federal del Trabajo Social No. 27.072/2014 se abrió todo un nuevo panorama para la formación, dado que, como indican Martínez y Agüero, la nueva ley “implica el inicio de un proceso de homogeneización en la formación y las prácticas profesionales, en un contexto de gran heterogeneidad y dispersión que ha existido históricamente en el Trabajo Social en Argentina y que en muchos casos no ha favorecido ni la calidad ni la competencia profesional, incluyendo iguales denominaciones de títulos para formaciones absolutamente disímiles y hasta antagónicas" (Martínez y Agüero, 2014, p. 91) .

En este marco de heterogeneidad seleccionamos la Facultad de Trabajo Social de la Universidad Nacional de Entre Ríos (UNER), porque ha sido una de las instituciones que ha desarrollado un proceso de reflexión sobre las distintas formas de enseñar la profesión y de reforma curricular que se remontan a mediados de los años noventa (Coria et al., 1999; De Jong, Ludi y Badano, 2003). La Licenciatura en Trabajo Social de la UNER es una carrera universitaria de grado, posee una duración de cinco años; el plan de estudios se conforma de un "Seminario de Ingreso", y dos ciclos: “Básico", de dos años de duración, y "Superior" 
Rivero-Rodríguez, Del Prado, Battilana-Amarilla y Coradini-Guilherme

de tres años de duración, los cuales contabilizan un proceso de enseñanza con 3.024 horas reloj. Se finaliza con la presentación y defensa de tesis. Las prácticas pre-profesionales se desarrollan en tercero y cuarto año.

La primera materia vinculada al Trabajo Social se dicta en el primer año de la carrera y se denomina "Historiografía de la Intervención Social" es de modalidad anual, obligatoria y posee una carga horaria total de 96 horas. Esta se encuentra íntimamente relacionada con la materia "Fundamentos de Trabajo Social", dictada de manera paralela.

Según el plan de estudios posee los siguientes contenidos mínimos:

Trabajo Social como disciplina y profesión de las ciencias sociales. Proceso de configuración histórico-social de la profesión. Diferentes matrices teóricas que participan de su proceso de configuración disciplinar. Trabajo Social en la relación Estado-Sociedad. Categorías centrales del Trabajo Social: sujeto-necesidades sociales- derechos-producción y reproducción social-. Condiciones materiales y simbólicas - Vida cotidiana. Una mirada histórica de estas categorías y sus resignificaciones en el recorrido de la disciplina.

Dicha asignatura integra el Área de Trabajo Social. El plan curricular se divide en diferentes áreas curriculares: Trabajo Social; Filosófica; Socio-histórica-antropológica; Salud; Investigación Social; Jurídico-político-económica; Pedagógica. El área de Trabajo social posee las siguientes materias: Historiografía de la Intervención Social; Trabajo Social, Modernidad y Cuestión Social; Trabajo Social y Configuración de Problemas Sociales; Trabajo Social y Vida Cotidiana; Intervención Profesional y Vida Cotidiana; Trabajo Social Contemporáneo; Intervención Profesional e Institucionalidad Social; Trabajo Social y Construcción Disciplinar; Seminario Interdisciplinario Familia; Problemática de lo Grupal, Organizacional e Institucional; Análisis Institucional y Organizacional.

La asignatura plantea como objetivo general: "Propiciar desde un análisis históricocrítico que los estudiantes comprendan las bases conceptuales sobre las que se construyen y fundamentan el Trabajo Social" (Resolución de Consejo Directivo No. 549/2018). Para el logro de dicho objetivo se divide en tres unidades: Unidad 1: Trabajo Social como disciplina de las ciencias sociales; Unidad 2: La configuración de Trabajo Social, y Unidad 3: Categorías principales en la trayectoria disciplinar.

La unidad 1 se subdivide en tema 1: "Imaginario social y ciencias sociales. El imaginario social y la profesión. El pensar como una opción potenciadora: los modos de pensar, diferentes claves de comprensión de lo social. La relación entre pensamiento, conocimiento y realidad"; y tema 2: "El surgimiento de las Ciencias Sociales en la Modernidad. Ciencias Sociales y Trabajo Social como profesión y disciplina. Matrices 
Rivero-Rodríguez, Del Prado, Battilana-Amarilla y Coradini-Guilherme

teóricas que participan del proceso de configuración disciplinar del Trabajo Social: Positivismo y Matrices Críticas. Visiones y/o tendencias en el Trabajo Social".

En el primer tema se analiza la noción de imaginario social, y su vínculo con la profesión. Es una unidad de carácter epistemológico en la que predominan textos de filósofos/as (Ester Díaz, Mario Heler, Hugo Zemelman) y reflexiones a partir de textos de Susana Cazzaniga, quien fuera docente de dicha casa de estudios en años previos, y directora de la Maestría en Trabajo Social. De un total de 13 textos, ocho pertenecen a la profesión y cinco son de filosofía.

La unidad 2, "La configuración del Trabajo Social", se encuentra dividida en dos temas; el primero se aboca en su totalidad a reflexiones históricas sobre la profesionalización del Trabajo Social; posteriormente, el segundo es el más amplio del programa y aborda la historia del Trabajo Social casi en su totalidad, trabajando 23 textos, entre los que se observan artículos de libros, revistas, leyes y tesis, es la parte más amplia del programa. Detallamos a continuación:

\section{Tema 1:}

“La acción social, organización y profesionalización de la asistencia. El Trabajo Social como práctica particular en la relación Estado-Sociedad y su vinculación con el proceso histórico. Las expresiones de la cuestión social y su papel en la configuración del Trabajo Social. Surgimiento del Trabajo Social en Europa y Estados Unidos. La propuesta teórica de Mary Ellen Richmond. El Caso Social Individual".

\section{Tema 2:}

“Emergencia del Trabajo Social en Latinoamérica. El Trabajo Social en la Argentina, la particularidad del contexto de surgimiento. La primera escuela: formación profesional y la intervención en la cuestión social. La intervención estatal durante la década del '40. Formación profesional y políticas desarrollistas. Críticas a la formación profesional: el Movimiento de Reconceptualización. La dictadura y su influencia en el desarrollo de la profesión. El retorno a la democracia y las principales líneas de desarrollo del Trabajo Social. Neoliberalismo: principales debates del Trabajo Social en Argentina".

La unidad 3, "Categorías principales en la trayectoria disciplinar" es la más breve del programa con ocho textos, de los cuales son el cincuenta por ciento de Trabajo Social y el otro cincuenta por ciento de filosofía.

“Categorías que estructuran el campo conceptual de Trabajo Social: Sujeto, Necesidades, Derechos, Producción y Reproducción Social, Condiciones materiales y simbólicas de vida, Vida Cotidiana. Las categorías y sus referencias en la teoría social, fundamentos de su pertinencia en Trabajo Social". 
Rivero-Rodríguez, Del Prado, Battilana-Amarilla y Coradini-Guilherme

En el programa se observa que se articulan textos y reflexiones desde el Trabajo Social en relación con la epistemología. Hay un conjunto de textos que realizan reflexiones sobre la historia de la profesión en un abanico de perspectivas -marxismo brasileño, trabajo social español, autores argentinos-, aunque con una impronta del Trabajo Social de la UNER Cazzaniga predominantemente y en menor medida otras docentes e investigadoras de la institución-. Se identifican también textos de las pioneras de la profesión, destacándose Mary Richmond: Diagnóstico Social y Caso Social Individual.

A la bibliografía se anexan leyes provinciales y nacionales, así como documentos de la Federación Argentina de Unidades Académicas de Trabajo Social (FAUATS).

\section{Brasil}

El contexto histórico de la institucionalización del Trabajo Social en Brasil se remonta a la década de 1930, en la que el Estado se ubica como agente de regulación social, a través de la ejecución de una protección social fragmentada orientada a la reproducción de la fuerza de trabajo. En este sentido, como aclaran Iamamoto y De Carvalho (2003), la primera Escuela Brasileña de Servicio Social fue fundada en 1936, a partir de una oferta privada vinculada a la Pontificia Universidad Católica de São Paulo -PUC / SP. En el período, la matriz rectora "del pensamiento y la acción del Trabajo Social emergente tiene su origen en la Doctrina Social de la Iglesia" (Yazbek, 2009b, p. 146). Esto significa que la profesión, en su génesis, se alinea con "posiciones humanistas conservadoras contrarias a las ideas liberales y marxistas, en la búsqueda de la recuperación de la hegemonía del pensamiento social de la Iglesia frente a la cuestión social" 5(Yazbek, 2009b, p. 146).

Sin embargo, vale la pena considerar que el Servicio Social Brasileño a lo largo de su historia ha experimentado reorientaciones en cuanto a las matrices teórico-metodológicas, con énfasis en la década de 1980, período en el que la teoría social de Marx comenzó a redimensionar la formación y el trabajo profesional. Esta teoría, como matriz teóricometodológica, parte de "un conocimiento que no es manipulador y que aprehende dialécticamente la realidad en su movimiento contradictorio" (Yazbek, 2009b, p. 151). Fruto de esta aprehensión crítica de la realidad, en la década de los noventa se realizaron varios debates dentro de la categoría profesional que culminaron en: a) el Código de Ética del Trabajador Social (Resolución CFESS No. 273/1993); b) en la Ley 8.662/1993 que establece el Reglamento de la Profesión (Conselho Federal de Serviço Social [CFESS], 2012); y, c) en las Directrices Generales del Currículo para los Cursos de Trabajo Social de la Associação Brasileira de Ensino e Pesquisa em Serviço Social [ABEPSS] (1996)

5 En sus múltiples manifestaciones la cuestión social constituye “a matéria prima e a justificativa da constituição do espaço do Serviço Social na divisão sociotécnica do trabalho e na construção/atribuição da identidade profissional" (Yazbek, 2009a, p. 129). 
Rivero-Rodríguez, Del Prado, Battilana-Amarilla y Coradini-Guilherme

Cabe destacar ciertos contenidos mencionados en los Lineamientos Curriculares como los principios que sustentan la formación profesional, entre ellos la "adopción de una teoría social crítica que posibilite la aprehensión de la totalidad social en sus dimensiones de universalidad, particularidad y singularidad" (ABEPSS, 1996, p. 6). Los Lineamientos también apuntan a un proyecto de formación profesional que presupone la formación teórico-metodológica, ético-política y técnico-operativa, es decir, la implementación de saberes inseparables, a través de Fundamentos: a) Núcleo de fundamentos teóricometodológicos de la vida Social; b) núcleo de los fundamentos de la particularidad de la formación sociohistórica de la sociedad brasileña; y, c) núcleo de los fundamentos del trabajo profesional.

El Trabajo Social en Brasil tiene una carrera única, la de Licenciado en Trabajo Social, tal grado en educación superior es otorgado por instituciones educativas a nivel de pregrado, y puede ser ofrecido por Instituciones de Educación Superior (IES) públicas o privadas. El título de Trabajador Social, en cambio, solo puede ser conferido por el Consejo Profesional6 de la región en la que el profesional se está capacitando.

A pesar de los avances logrados, actualmente se imponen desafíos en el ámbito de la formación en Trabajo Social, con énfasis en la heterogeneidad de la modalidad de oferta de los Cursos de Grado, que involucra tanto la docencia presencial como la Educación a Distancia (EAD)7 7 . Es posible evidenciar que en 2018 existen 480 Cursos de Grado en Trabajo Social en Brasil -que involucran tanto la modalidad presencial como la modalidad a distancia-: $85,42 \%$ en Instituciones de Educación Superior privadas y 14,58\% en Instituciones de Educación Superior públicas (Instituto Nacional e Estudos e Pesquisas Educacionais Anísio Teixeira [INEP], 2019).

Según Conselho Federal de Serviço Social [CFESS] (2020) llegamos a 190.000 Trabajadores Sociales registrados en todo el país, el número exponencial de profesionales y escuelas de Trabajo Social apunta a una diversidad de formatos en los currículos escolares, que siguen, o no, los Lineamientos Curriculares de ABEPSS. Los Cursos de Trabajo Social deben tener al menos 2.700 horas, con una duración de cuatro años (8 semestres), y al menos el 15\% de la carga de trabajo debe estar cubierto por la pasantía supervisada obligatoria entre 2 o 3 semestres (ABEPSS, 1996).

En cuanto al objetivo de este estudio, considerando el espacio geográfico brasileño, para el análisis se definieron como muestra dos escuelas de Trabajo Social, que cumplen con

6 La habilitación de Trabajador Social es emitida por los Consejos de Servicio Social a nivel regional, el CRESS, los cuales están orgánicamente vinculados al Consejo Federal de Servicio Social - CFESS. En Brasil tenemos un total de 27 consejos regionales, uno por cada estado brasileño.

7 Enseñanza a Distancia - EAD en Brasil, denominados cursos On Line en otros países 
Rivero-Rodríguez, Del Prado, Battilana-Amarilla y Coradini-Guilherme

los siguientes criterios: a) ser una universidad8; b) observar la naturaleza contemplando 1 ( uno) público y 1 (uno) privado; c) tener su sede en el estado de Rio Grande do Sul-RS; y, d) ser las primeras instituciones educativas en ofrecer el curso de Trabajo Social -en su carácter institucional- en Rio Grande do Sul - RS. Con base en estos criterios, constituyen la muestra: a) Pontificia Universidad Católica de Rio Grande do Sul - PUC / RS, primera Institución de Educación Superior - IES privada, en ofrecer el curso de Trabajo Social en RS, desde 1945. b) Universidad Federal de Pampa - UNIPAMPA, la primera IES pública en ofrecer el curso de Servicio Social en RS, desde 2006.

Como primer punto de análisis, se puede destacar que la formación pionera en Trabajo Social en RS es responsabilidad del ámbito privado. "El Curso de Servicio Social de UNIPAMPA inició sus actividades el 16 de octubre de 2006. Es el primer curso público en una Universidad Pública Federal del Estado de Rio Grande do Sul" (UNIPAMPA, PPC, 2016: 35). Considerando el cronograma de creación del Curso de Trabajo Social en las Instituciones de Educación Superior-IES que componen la muestra (1945 y 2006), son más de 60 años de formación en el ámbito privado antes de ofrecer un curso público que cumpla con los supuestos de la educación superior como derecho constitucional. A partir de estas dos instituciones, considerando el objetivo del estudio en cuestión, las materias impartidas en el primer año de formación $\left(1^{\circ}\right.$ y $2^{\circ}$ semestres) se definieron como un recorte para el análisis, señalando así a los autores y temáticas que acercan a los estudiantes cuando ingresan a los cursos de Trabajo Social.

Es importante resaltar que los dos cursos que componen la muestra siguen los Lineamientos Curriculares ABEPSS, que ya apuntan a un currículo acorde con la perspectiva teórica de la profesión en Brasil, siguiendo una perspectiva crítica. En el conjunto de asignaturas y autores de los dos currículos durante los primeros contactos con la profesión (en el primer año de formación) se hace énfasis en el conocimiento inicial sobre la profesión y elementos de conformación social, las asignaturas ofertadas se pueden dividir en dos grupos:

a. El primer grupo trata los temas de estudio que son la base de los fundamentos de la formación en Trabajo Social, en lo que respecta a la implantación del Trabajo Social. Este grupo comprende los siguientes componentes curriculares: Introducción al Trabajo Social; Asuntos sociales y laborales; Construcción social del sujeto; Sociología; Formación sociohistórica y servicio social; Sociedad capitalista y cuestión social; Ética y Ciudadanía (1er semestre). Entornos laborales y de servicios sociales; Constitución de la Identidad del Trabajador Social; Estado, Política Social y Sistema de Protección Social; Fundamentos del Trabajo Social I; Laboratorio de formación profesional; Proyecto de Servicio Social y Ético-Político;

8 La Universidad en Brasil tiene como premisa no disociar la formación del trípode: enseñanza, investigación y extensión, fortaleciendo así el proceso de formación en el nivel superior. 
Rivero-Rodríguez, Del Prado, Battilana-Amarilla y Coradini-Guilherme

Protección social; Derecho y Legislación Social; Economía política; Fundamentos Históricos y Teórico-Metodológicos del Trabajo Social I; Fundamentos ontológicos del ser social ( $2^{\circ}$ semestre).

b. El segundo grupo se refiere a temas interdisciplinarios. Cabe destacar el esfuerzo en cuanto a la agrupación de asignaturas, sin embargo, el trabajo de análisis demuestra la inseparabilidad entre las asignaturas, la totalidad de las asignaturas identificadas engloban un conjunto de conocimientos y habilidades demarcados por ABEPSS (1996) como temas de estudio para la formación y la práctica preprofesional, basada en los siguientes componentes curriculares: Fundamentos de Psicología; Lectura y producción de textos; Laboratorio de Producción Documental en Servicio Social I; Identidades culturales (1er semestre). Introducción a los Derechos Humanos ( $2^{\circ}$ semestre).

Sobre los autores y trabajos utilizados en las bibliografías obligatorias, los/las autoras y las obras se pueden agrupar de diferentes formas. Se eligió el orden cronológico, llamándolos clásicos y contemporáneos, estos últimos se expresan en una producción intelectual más reciente con producciones nacionales e internacionales. Esta organización permite comprender el nivel de aproximación de los estudiantes de Trabajo Social de ambos cursos a las obras clásicas del pensamiento crítico. Es evidente, en esta forma de organización, que el trabajo de Karl Marx está presente en el proceso de formación desde el inicio de la graduación en Trabajo Social, siguiendo la perspectiva adoptada en la formación brasileña. Además de Marx, durante la formación en UNIPAMPA se identificaron obras de August Comte, Émile Durkheim, Friedrich Engels y Max Weber, de acuerdo con los lineamientos de las Directrices Curriculares de la ABEPSS, que apuntan a las matrices clásicas del pensamiento sociológico como base para la formación (ABEPSS, 1996).

Entre las obras y autores contemporáneos, destaca el uso de referencias reconocidas internacionalmente, como Mészaros, Huberman, Harvey, Castel y Heller. La formación en los dos cursos estudiados está anclada en obras contemporáneas, especialmente obras y autores de renombre internacional, que consolidan la producción teórica del Trabajo Social Brasileño como: Iamamoto, De Carvalho, Netto, Yazbek, Martinelli, Behring, Boschetti, Couto, Faleiros, Raichelis.

En cuanto al objetivo inicial de evaluar el acceso de los estudiantes a las obras clásicas, se puede decir que en los cursos analizados, las obras sirvieron de referencia obligada a una formación que ofrece, aunque más tímidamente, el acceso a los clásicos.

La mayoría de las obras contemporáneas, especialmente en el área de Sociología y Trabajo Social, van desde los consagrados autores del Trabajo Social Brasileño hasta los jóvenes investigadores que aparecen en la escena nacional. 


\section{Paraguay}

En Paraguay la historia del Trabajo Social se remonta a 1939, con la fundación de la Escuela de Visitadoras Polivalentes de Higiene (García, 1996), el contexto de emergencia y de institucionalización de la profesión se produce a partir de acontecimientos y sucesos vinculados con el desarrollo de la sociedad civil y el Estado configurado por expresiones muy particulares del país, en sus dimensiones económica, política, social. Paraguay, con una economía dependiente, basado en el modelo agroexportador, régimen político sustentado en la dictadura militar por 35 años, determinando el bajo nivel de participación ciudadana, aspectos que han estructurado al Paraguay y al Trabajo Social en particular.

La carrera de Trabajo Social ha transitado de su condición de tecnicatura a profesión universitaria en 1963, siendo la única institución formadora de Trabajadoras/es Sociales en ese entonces, pasando a depender de la Universidad Nacional de Asunción (UNA) ${ }^{9}$. Actualmente la carrera se imparte en cuatro universidades públicas y cuatro privadas.

La primera carrera universitaria de Trabajo Social se inicia en la Universidad Nacional de Asunción (UNA) en 1963. Ese año se da el traslado del Instituto Dr. Andrés Barbero, que dependía del Ministerio de Salud Pública y Bienestar Social, institución que impartía cursos de Servicio Social, Enfermería y Obstetricia, siendo este proceso resultado de las exigencias de la época y de la imperiosa necesidad de contar con una carrera universitaria de Servicio Social para la atención de las demandas de la ciudadanía en ese período.

García (2005) expresa que en esa década el incremento de instituciones del Estado que atendían los llamados problemas sociales vía servicios sociales públicos, requería profesionales con niveles de formación universitaria; de esta forma, el Servicio Social acompañaba esa nueva demanda.

En el plan curricular de la carrera de Servicio Social de 1963 de la UNA se entendía que:

El Servicio Social se define como un arte y una ciencia; el profesional competente realiza su labor basada en los conocimientos científicos de la sociedad y del comportamiento humano y hace uso de la habilidad adquirida en el arte de la práctica. El objetivo de una escuela es impartir los

9 Posteriormente se crea la carrera de Trabajo Social en la Facultad de Derecho, Ciencias Políticas y Sociales de la Universidad Nacional de Pilar, y en el periodo del 2000 surgen otras carreras en el ámbito público como privado, en la Facultad de Humanidades y Ciencias de la Educación de la Universidad Evangélica del Paraguay; en la Facultad de Trabajo Social de la Universidad Técnica de Comercialización y Desarrollo (UTCD); en la Facultad de Ciencias Jurídicas, Humanas y Sociales de Universidad Autónoma de Encarnación; en la Facultad de Humanidades, Ciencias Sociales y Cultura Guaraní de la Universidad Nacional de Itapúa; en la Facultad de Humanidades y Ciencias de la Educación de la Universidad Nacional de Concepción; en la Facultad de Ciencias Sociales y Humanidades de la Universidad Politécnica y Artística del Paraguay. 
conocimientos y ayudar al estudiante a desarrollar la destreza que conjuntamente completa el arte y la ciencia de la profesión. (García, 2005, p. 275)

Durante largos períodos la carrera ha estado tutelada por organizaciones de formación de los Estados Unidos y agencias de cooperación extranjera, quienes dieron el apoyo para el inicio de la carrera en la Universidad (García, 2005), y es en 1989, tras la caída de la dictadura del general Alfredo Stroessner, cuando se produce un cambio curricular en la carrera de Trabajo Social de la UNA (Resolución No. 2832-00-89). Esta nueva malla curricular no expresa de manera explícita un concepto de Trabajo Social, tanto el perfil profesional como los objetivos de la carrera están orientados a un profesional que interviene en los problemas sociales a través de los llamados métodos tradicionales -caso, grupo y comunidad-. La teoría social está presente para definir más bien el rol del Trabajo Social y no para orientar la formación, y la práctica profesional se enmarca en la implementación de técnicas de intervención según los niveles de profundización. Un avance importante fue la incorporación de la investigación como parte del perfil, así como también un énfasis en la pasantía profesional (Battilana y López, 2018).

En la carrera de Trabajo Social de la UNA a partir del cambio curricular en 2001 se adopta la siguiente idea:

Es una profesión universitaria que interviene en los problemas sociales, utilizando los aportes de las diversas ciencias sociales y buscando revertir una situación problemática en alternativas de solución, utilizando los recursos de la comunidad, de instituciones u organizaciones y los conocimientos, destrezas y habilidades del profesional. Esto significa que en la intervención del Trabajo Social se articula la teoría con la práctica. (UNA, 2011 citado en Battilana y López, 2018, p. 42)

Con el plan curricular de 2001 se instala el debate de las dos perspectivas en Trabajo Social; la primera que entiende la profesión como la evolución de las primeras formas de ayuda, la endógena; y la otra que ubica a la profesión como producto de un proceso histórico-crítico, siendo las políticas sociales y el propio Trabajo Social como dispositivos que deben generar las respuestas a la cuestión social (Montaño, 2000).

La perspectiva central en el proceso de formación en Trabajo Social de la UNA coloca la necesidad de entender que la materia prima de la profesión son las expresiones de la cuestión social y que las políticas sociales configuran el espacio socio-ocupacional del Trabajo Social.

Es así que el proyecto académico en lo que va de 2001 hasta la última aprobación de la malla curricular en 2015 en la UNA, continúa en el plan curricular la perspectiva teórica histórica-crítica, buscando la superación del pragmatismo, del accionar basado en el sentido común o los simples voluntarismos, y pretende incorporar la investigación como eje 
Rivero-Rodríguez, Del Prado, Battilana-Amarilla y Coradini-Guilherme

estructurante de la intervención profesional, siendo áreas fundamentales a ser trabajadas en la formación; la teoría social, el énfasis en la realidad, y los conocimientos y fundamentos del Trabajo Social, claves para toda intervención profesional fundada.

En la última década se han observado "avances significativos en el proceso de la formación profesional en cuanto a la incorporación del análisis de la historia vinculada al contexto, buscando situar al Trabajo Social en la perspectiva histórica-crítica, siendo una primera búsqueda de superación teórico-metodológica con la profesión enmarcada en el enfoque tradicional-endogenista" (Battilana y López, 2018, p. 50).

Con la implementación de los últimos planes curriculares, la necesidad de seguir profundizando en el análisis y comprensión de la "cuestión social" como materia prima del Trabajo Social, es trabajada por un grupo de docentes en la asignatura del primer año de la carrera, denominada Práctica Pre Profesional a partir del año 2006, “buscando tensionarla con la realidad para aprehender el funcionamiento de la sociedad capitalista, tomando no solamente la dimensión de las desigualdades que las producen sino de las resistencias que desarrollan los sujetos" (Battilana y López, 2018, p. 50).

El ideario de Trabajo social que en su búsqueda de ruptura con enfoques endogenistas, hoy se acerca al concepto de:

Trabajo Social y Servicio Social es una profesión que se inserta en el ámbito de las relaciones entre sujetos sociales y entre estos y el Estado, en los distintos contextos socio históricos de actuación profesional. Desarrolla una praxis social y un conjunto de acciones socioeducativos, que inciden en la reproducción material y social de la vida con una perspectiva de transformación social comprometida con la democracia y el enfrentamiento de las desigualdades sociales, fortaleciendo la autonomía, la participación y el ejercicio de la ciudadanía, en la defensa y conquista de los derechos humanos y la justicia social. (Comité Latinoamericano y Caribeño de Organizaciones Profesionales de Trabajo Social/Servicio Social [COLACATS], 2012) ${ }^{10}$

La carrera de Trabajo Social en la UNA se configura por áreas de trabajo: A) teoría social, nuclea a las disciplinas de las ciencias sociales y humanas que se apoyan en la construcción del pensamiento teórico. En esta área el eje está colocado en la preparación teórica, desarrollando la postura investigativa que integra el hacer profesional. El conjunto de conocimientos teóricos permite develar la realidad social. B) Procesos sociales y realidad nacional, se analiza la dinámica, el movimiento interno de la sociedad, comprendiendo la constitución y articulación de los diferentes actores sociales y sus luchas. Es clave el estudio y análisis de partidos políticos, movimientos sociales, sector empresarial y el Estado. C) Conocimientos conexos para la intervención, integra las bases para el desarrollo del trabajo científico y para la constitución de una intervención fundada.

10 Concepto de Trabajo Social planteado por el Comité Latinoamericano y Caribeño de Organizaciones Profesionales de Trabajo Social/Servicio Social (COLACATS, 2012). 
En la formación de Trabajo Social en la UNA las asignaturas clave son:

- Fundamentos del Trabajo Social, donde se desarrolla la historia y la teoría que han sustentado los diferentes modelos o propuestas teóricas y metodológicas del Trabajo Social y otorga la base para la implementación de una intervención profesional fundada. Durante los cuatro años de formación se desarrolla esta asignatura, siendo los contenidos principales: institucionalización del Trabajo Social, perspectivas de la profesión, proceso histórico de Trabajo Social, debates post reconceptualización y contemporáneos, aportes del Trabajo Social en la construcción de proyectos societarios, entre otros.

- Abordaje profesional -práctica- es la materia troncal en todo el proceso de formación del Trabajo Social en la UNA, lo cual es clave al ser una profesión predominantemente interventiva. La práctica se desarrolla en espacios socioocupacionales, que garantizan un proceso pedagógico donde se construye la propuesta de estrategias de intervención profesional.

El plan curricular de la carrera de Trabajo Social de la Universidad Nacional de Asunción, que se desarrolla desde 2016, es en la modalidad semestral con 3.330 horas, equivalente a 222 créditos, con asignaturas del área de las ciencias sociales y humanas como son: Epistemología de las Ciencias Sociales, Teoría sociológica clásica, Teoría sociológica contemporánea, Historia de las transformaciones mundiales, Economía, Psicología general, Psicología social, Teoría política I y II, entre otros; asignaturas del área de los procesos sociales como Realidad paraguaya, Política social y Trabajo Social I, II, Política social en Paraguay, Formación sociohistórica de la sociedad paraguaya I y II, Problemáticas sociales contemporáneas, entre otros. Así también asignaturas instrumentales como Comunicación oral y escrita, Gestión social, Investigación, y otros; y las asignaturas troncales por un lado los Fundamentos del Trabajo Social, Cuestión Social y Trabajo Social y el área práctica que son a través de la asignatura de Abordaje Profesional, y se culmina la carrera con la presentación de un trabajo final de grado (tesis). Estas asignaturas responden al perfil de egreso que pretende formar un profesional que comprenda la realidad social a partir de categorías analíticas de la teoría social, así como las particularidades de Paraguay inserto en los procesos regionales y mundiales, para una sólida intervención profesional, acorde a las demandas ciudadanas. Con capacidad de elaborar propuestas de intervención profesional que promuevan el ejercicio de los derechos humanos, teniendo como fundamento la comprensión de las relaciones de clase, género, generación e interculturalidad. Contribuyendo desde la disciplina a fortalecer un diálogo igualitario y sostenido para intervenciones fundamentadas en equipos de trabajo interdisciplinarios. Reconociendo a los sujetos con sus saberes, subjetividades, historicidades estableciendo diálogos que conduzcan a la construcción de relaciones emancipatorias, $y$, finalmente, un profesional que asuma los valores de respeto, libertad, justicia social, solidaridad y participación 
Rivero-Rodríguez, Del Prado, Battilana-Amarilla y Coradini-Guilherme

consensuados por el colectivo profesional a nivel de la región del Cono Sur (Resolución No. 0183-00-2015).

Haciendo un recorrido por los planes curriculares de la carrera de Trabajo Social en la UNA se observa cómo la asignatura introductoria a la disciplina (Ver Tabla 1) ha sufrido modificaciones en su denominación, dando una idea de la perspectiva de la misma.

Tabla 1. Introducción a la disciplina según Plan de Estudios.

\begin{tabular}{|c|c|c|c|c|}
\hline Plan 1963 & Plan 1988 & Plan 1989 & Plan 2001 & Plan 2016 \\
\hline $\begin{array}{c}\text { Introducción } \\
\text { al Servicio } \\
\text { Social }\end{array}$ & $\begin{array}{c}\text { Trabajo } \\
\text { Social I y II }\end{array}$ & $\begin{array}{c}\text { Introducción } \\
\text { al Trabajo } \\
\text { Social }\end{array}$ & $\begin{array}{c}\text { Fundamentos } \\
\text { históricos, } \\
\text { teóricos y } \\
\text { metodológicos del } \\
\text { Trabajo Social I }\end{array}$ & $\begin{array}{c}\text { Fundamentos } \\
\text { del Trabajo } \\
\text { Social I }\end{array}$ \\
\hline
\end{tabular}

Fuente: elaboración propia

Ahora bien, analizando la asignatura de "Fundamentos del Trabajo Social" del último plan curricular de la carrera, la misma se desarrolla en cuatro semestres, iniciando con "Fundamentos del Trabajo Social I", en el segundo semestre de la carrera en el primer año de cursada. Tiene como objetivo general: "Lograr que los y las estudiantes conozcan la historia del Trabajo Social, el origen y los procesos de institucionalización de la profesión". El programa de la asignatura está distribuido en tres unidades:

Unidad 1. Siendo el eje central de los contenidos El Trabajo Social en la división social de trabajo. La cuestión social y la institucionalización del Trabajo Social, se requiere contextualizar el modelo de producción capitalista, sus retraimientos y el ascenso de la clase obrera, como determinantes del origen de la profesión (Trabajo Social). Contextualizar el siglo XX y las principales expresiones de la cuestión social, la racionalización de la práctica de la asistencia y por ende la creación de las escuelas de profesionalización de Servicio Social, comprender la tradición del Servicio Social en la perspectiva europea, norteamericana y la influencia de la Iglesia.

Unidad 2. Los contenidos centrales, El significado de los servicios sociales y la dimensión técnico-política ${ }^{11}$ de la profesión. Particularidades y espacios de actuación profesional. Las corrientes que explican el origen y la institución del Trabajo Social: perspectiva endogenista y la histórica crítica, analizando sus precursores, contexto

11 En el programa figura "técnico-político", aunque en este punto, se desarrolla la dimensión ético-política de la profesión, y la necesidad de contar con competencias teóricas, técnicas y ético-políticas. 
Rivero-Rodríguez, Del Prado, Battilana-Amarilla y Coradini-Guilherme

sociopolítico y económico que las determina. Conceptualizaciones de la Cuestión Social, la Exclusión Social, la Vulnerabilidad y los criterios de pobreza en la perspectiva crítica.

Unidad 3. Y los ejes centrales son las organizaciones de Trabajo Social en Paraguay y los procesos de legislación profesional del Trabajo Social. Introducción a la ética en Trabajo Social: Conceptos, definiciones y funciones asignadas al Trabajo Social en distintos contextos y momentos. Ubicación del Trabajo Social en la complejidad de lo social. Conceptos, definiciones y funciones de Trabajo Social en la perspectiva endogenista y en la perspectiva histórico-crítica. Revisión de la definición de Trabajo Social de la Federación Internacional (FITS). Revisión de la definición de Trabajo Social y del Comité Mercosur de Organizaciones de Trabajo Social. Participación de Paraguay en dicho Comité. Trabajo Social en Paraguay; bases para un análisis sobre su génesis y proceso de institucionalización.

Las referencias bibliográficas utilizadas en la asignatura han sido organizadas con textos del área de Trabajo Social tanto latinoamericano como nacional. Si bien Paraguay aún no tiene mucha producción, los aportes de Stella García han marcado en la formación del Trabajo Social paraguayo, como así también autores y trabajadores sociales brasileños que desde la década de los años 80 han generado producción en la línea de la perspectiva histórica crítica como María Lucía Martinelli, Marilda Iamamoto, Carlos Montaño, y autores argentinos que tienen experiencia y producción teórica en la disciplina, como Susana Cazzaniga y Alfredo Carballeda.

\section{Uruguay}

En Uruguay existen dos formaciones en Trabajo Social: una propuesta corresponde a la oferta pública de la Universidad de la República; la otra corresponde a la oferta privada de la Universidad Católica del Uruguay.

En términos generales, observamos que "tanto la bibliografía como diferentes investigaciones realizadas en relación al proceso de origen e institucionalización del Trabajo Social en Uruguay, hablan de la fuerte asociación de las ideas higienistas en el surgimiento del Servicio Social en Uruguay (en 1923 se crea la primera escuela de Visitadoras Sociales de Higiene en la órbita del Instituto de Higiene Experimental, de la Facultad de Medicina), lo que permite visualizar un origen profesional fuertemente condicionado al surgimiento del Estado benefactor y su tratamiento de la llamada cuestión social" (Rivero, 2018, p. 20).

La formación a nivel privado se inicia con la Escuela de Servicio Social del Uruguay (ESSU), creada con el apoyo de la Unión Católica Internacional de Servicio Social, que en la década de los años 80 del Siglo XX, pasa a formar parte de la Universidad Católica del Uruguay (Bralich, 1993). 
Rivero-Rodríguez, Del Prado, Battilana-Amarilla y Coradini-Guilherme

A nivel de la enseñanza pública, en 1954 se crea la formación profesional en el ámbito universitario a través de la Escuela Universitaria de Servicio Social (EUSS) que emite el título de Asistente Social Universitario.

A principios de la década de los años 90 (S. XX) se crea la Facultad de Ciencias Sociales en el marco de la Universidad de la República. En esta nueva institucionalidad se incluye la Licenciatura en Trabajo Social, junto a las Licenciaturas en Sociología, Ciencias Políticas y posteriormente en Desarrollo.

Según Rivero (2018, p. 25) “esta nueva institucionalidad supuso un desafío importante para el Trabajo Social ya que su perfil profesional, básicamente técnico, se ve interpelado al incluirse en una Facultad donde las otras Licenciaturas forman intelectuales". Acosta (2016) se refiere a esta integración a la Facultad de Ciencias Sociales como:

la expresión de una voluntad por parte de Trabajo Social para alcanzar un nuevo nivel en el proceso de maduración política y académica, pasando de ser un técnico para ser un intelectual (...) en un intento de mantener una relación entre iguales con las otras ciencias sociales. (Acosta, 2016, p. 40).

Hasta la fecha se implementaron dos planes de estudio: Plan 1992 y Plan 2009. Este último es el actualmente vigente, la malla curricular de la carrera consta de 360 créditos (4 años y la monografía final) dividido en dos ciclos: Inicial y Avanzado ${ }^{12}$.

El Ciclo Inicial representa 120 créditos de los 360 que componen las distintas Licenciaturas. Tiene una duración de tres semestres: el primer semestre contiene asignaturas obligatorias, mientras que los siguientes semestres tienen tanto actividades obligatorias como optativas.

Se imparten asignaturas de formación general ya que aún el estudiante no ha optado (formalmente) por la Licenciatura que cursará.

En este Ciclo Trabajo Social ofrece una asignatura obligatoria “Cuestión social en la historia (ocho créditos); una asignatura electiva ${ }^{13}$ Introducción al Trabajo Social (diez créditos) y dos optativas (una metodología de investigación y otra sobre políticas sociales).

En el Ciclo Avanzado (240 créditos) es donde se aprenden los contenidos específicos de la profesión, dicho trayecto se encuentra dividido en ocho módulos, los cuales agrupan distintas asignaturas:

12 Plan de estudio 2009 Facultad de Ciencias Sociales https:/ / cienciassociales.edu.uy/plan-de-estudios-2009/

13 Es electiva ya que las y los estudiantes tienen que elegir obligatoriamente dos asignaturas de una oferta de cuatro. Cada Licenciatura presenta una asignatura introductoria. 
Rivero-Rodríguez, Del Prado, Battilana-Amarilla y Coradini-Guilherme

Módulo 1: Fundamentos Teórico-Metodológicos del Trabajo Social, 81 créditos.

Módulo 2: Políticas Públicas, Planificación y Gestión, 30 créditos.

Módulo 3: Componentes Psicosociales de la Intervención Profesional, 14 créditos.

Módulo 4: Teorías Sociales, 30 créditos.

Módulo 5: Metodología de la Investigación, 27 créditos.

Módulo 6: Introducción al Pensamiento Filosófico, 16 créditos.

Módulo 7: Análisis de los Procesos Históricos, 12 créditos.

Módulo 8: Trabajo Final, 30 créditos.

El módulo 1. "Fundamentos teóricos metodológicos del Trabajo Social” incluye las siguientes asignaturas semestrales: "Trabajo Social I", "Trabajo Social II", "Trabajo Social III" y "Trabajo Social IV"; que corresponderían a las asignaturas de contenido teórico, e "Introducción a Proyectos Integrales", "Proyectos Integrales I" y "Proyectos Integrales II"; materias que constituirían las prácticas preprofesionales. También se incluye en dicho módulo la materia "Laboratorio" -orientado al estudio de técnicas-.

Los Proyectos Integrales se desarrollan en cuatro semestres y en los mismos se realiza la práctica preprofesional. El estudiante puede elegir entre cuatro opciones, cada una con su programa y su bibliografía específica.

A modo de ejemplo de cómo se ha desarrollado la formación específica se presenta el contenido del Área Trabajo Social en los diferentes planes de estudio.

Tabla 2. Formación específica de la disciplina según planes de estudio.

\begin{tabular}{|c|c|c|c|c|}
\hline AÑOS & PLAN 67 & PLAN 67 (R87) & PLAN 92 & PLAN 2009 \\
\hline 1 & $\begin{array}{l}\text { Trabajo } \\
\text { Social }\end{array}$ & Trabajo Social & Trabajo Social & $\begin{array}{l}\text { Cuestión Social en la historia (Primer } \\
\text { semestre Ciclo Inicial) }\end{array}$ \\
\hline 2 & Caso & $\begin{array}{l}\text { Metodología de } \\
\text { Trabajo Social }\end{array}$ & $\begin{array}{l}\text { Metodología de } \\
\text { Intervención } \\
\text { Profesional (MIP) } \\
1 \text { Nivel macro }\end{array}$ & $\begin{array}{l}\text { Introducción Trabajo Social (Tercer semestre } \\
\text { Ciclo Inicial) } \\
\text { Trabajo Social 1(Cuarto semestre, Ciclo } \\
\text { Avanzado) } \\
\text { Laboratorio (Cuarto semestre, ciclo } \\
\text { avanzado), } \\
\text { Introducción a los Proyectos Integrales } \\
\text { (cuarto semestre, ciclo avanzado) }\end{array}$ \\
\hline 3 & Grupo & Talleres & MIP 2 Nivel meso & $\begin{array}{l}\text { Trabajo Social II (quinto semestre); } \\
\text { Trabajo Social III (sexto semestre) } \\
\text { Proyecto Integral I (quinto y sexto semestre) }\end{array}$ \\
\hline 4 & Comunidad & Talleres & MIP 3 Nivel micro & $\begin{array}{l}\text { Trabajo Social IV (séptimo semestre) } \\
\text { Proyectos Integral II (séptimo y octavo } \\
\text { semestre) }\end{array}$ \\
\hline 5 & - & Talleres & -- & Monografía \\
\hline
\end{tabular}

Fuente: elaboración propia. 
Rivero-Rodríguez, Del Prado, Battilana-Amarilla y Coradini-Guilherme

En función del objetivo de este trabajo nos detendremos en el análisis de la asignatura Introducción al Trabajo Social14. Esta asignatura tiene una carga horaria de 75 horas en un semestre, que se distribuye en un espacio teórico y un espacio práctico. El contenido se orienta en dos grandes módulos: en el primero se trabaja el concepto de intervención social y en el segundo la intervención profesional.

El módulo uno analiza las características de las estrategias de intervención en general y en particular en actores sociales relevantes: la Iglesia, organizaciones filantrópicas, el Estado, y los organismos internacionales. Incluye, a su vez, el análisis de los objetivos de la intervención social, entendiendo como principal finalidad la cohesión social y específicamente la orientación de estrategias en tres dimensiones: económica (disciplinamiento y control social), política (construcción de ciudadanía) y social (inclusión social).

El segundo módulo trabaja la temática de intervención profesional partiendo de analizar las modalidades tradicionales de intervención en Trabajo Social que, si bien presenta un desarrollo histórico, se enfoca desde una perspectiva de determinantes teóricometodológicos de mayor influencia en el contexto del Uruguay. A este análisis se incorpora la crítica a las modalidades tradicionales realizada por Paulo Netto, trabajando el concepto de sincretismo en Trabajo Social. En un segundo momento se trabaja el Movimiento de Reconceptualización y las actuales modalidades de intervención desarrolladas a nivel regional, específicamente la propuesta de Margarita Rozas. Finaliza el módulo con el análisis del proyecto ético-político y su relevancia para el desempeño profesional.

El análisis de la asignatura nos permite observar que se introduce al estudiante en la disciplina de Trabajo Social desde una perspectiva general (intervención social) para luego centrarse en la intervención profesional.

En relación a la bibliografía cada módulo presenta una lógica diferente. En el primero se articulan autores provenientes de la Sociología, Ciencia Política, Filosofía y de Trabajo Social. En tanto, el segundo módulo presenta una bibliografía específica de Trabajo Social donde tienen un peso relevante los autores brasileños, aunque también se observa bibliografía de autores argentinos y uruguayos.

\section{Conclusiones}

En este trabajo analizamos las asignaturas introductorias, las cuales funcionan como puerta de entrada a la disciplina, muestran las características específicas de la profesión así como el diálogo permanente con las ciencias sociales. Este enfoque complejiza la formación

14 https://cienciassociales.edu.uy/wp-content/uploads/2019/12/Introducc-Trabajo-Social-Edicion2019.pdf. 
Rivero-Rodríguez, Del Prado, Battilana-Amarilla y Coradini-Guilherme

y muestra una perspectiva en la que pierde peso la instrumentalidad para ir consolidando una formación centrada en una visión teórica-metodológica y ético-política de la disciplina. Es decir, perspectivas que hacen énfasis en las técnicas, que centran su accionar en la pregunta sobre ¿Cómo se interviene? sin poner énfasis en el conjunto de preguntas que refieren a ¿Para qué? ¿Con quiénes? y ¿Desde dónde?

La formación en Trabajo Social requiere no sólo las asignaturas específicas de la disciplina, en todas las unidades analizadas la formación implica una articulación con el conjunto de teorías sociales clásicas y contemporáneas de las ciencias sociales. Es decir, que se ha desarrollado una complejización de la formación en un proceso histórico del cual uno de los mojones relevantes fue el debate establecido por el Movimiento de Reconceptualización.

En las asignaturas analizadas se observa un predominio del enfoque crítico en Trabajo Social. Según Rivero y Vecinday (2005) este enfoque emerge de las posiciones generadas a partir del Movimiento de Reconceptualización, el cual surge en América Latina entre la década del ‘60 y la década del ‘80. Los debates presentes en este movimiento dan cuenta de un proceso complejo y contradictorio que implica importantes cambios al interior del Trabajo Social. Quizás uno de los más relevantes sea que supuso una ruptura con el Servicio Social tradicional.

Podemos identificar dos grandes líneas: la tecnocrática y la científica. Estas dos vertientes centralizaron la discusión acerca de cómo se identificaba la profesión: era una técnica o era una ciencia. El posicionamiento frente a esta discusión hace que el enfoque de formación sea muy diferente, esto se observa claramente al analizar las currículas de las unidades académicas. En este sentido, todas las asignaturas analizadas presentan una orientación hacia una visión científica y dentro de esta línea un enfoque crítico.

A pesar de haber incluido en el análisis pocas unidades académicas, podemos aventurar que el enfoque crítico predomina en estos cuatro países del cono sur latinoamericano.

En general la bibliografía utilizada se concentra en dos vertientes: a) en lo que respecta a las Ciencias Sociales se trabajan autores clásicos y contemporáneos con gran variedad de orientaciones; b) en cuanto a Trabajo Social se observa que los/las autores/as son, en su mayoría, provenientes del cono sur latinoamericano, especialmente Brasil y Argentina.

Con relación a la bibliografía, el formato utilizado es libro o artículo de revista, así como documentos. No identificamos la utilización de diccionarios y manuales de Trabajo Social, formato que es utilizado frecuentemente en otras latitudes. A nuestro entender, esto 
Rivero-Rodríguez, Del Prado, Battilana-Amarilla y Coradini-Guilherme

señala la diversidad teórica para entender las diferentes dimensiones de la disciplina y es coherente con una formación desde la perspectiva del pensamiento crítico.

Para finalizar, creemos que realizar una reflexión que abarque diferentes países de la región es una apuesta por seguir pensando en conjunto y construyendo un Trabajo Social Latinoamericano, intentando recuperar en ello la tradición y la perspectiva política de la tradición que recupera lo mejor de la Reconceptualización, proceso que fue fruto de un contexto de luchas populares contra la injusticia social y por la plena vigencia de los derechos humanos.

Por tanto, consideramos que el presente trabajo es un aporte para continuar pensando y reflexionando sobre cómo enseñamos la profesión, cuáles son los primeros contactos que tienen los estudiantes con el Trabajo Social. Reflexión que va de la mano con el conocimiento sobre los estudiantes, sus representaciones sociales acerca del rol del trabajo social en el contexto actual, sus formas de habitar la universidad, relacionarse con el conocimiento y el hecho de ser joven hoy.

\section{Referencias bibliográficas}

Acosta, L. (2016). El proceso de renovación del Trabajo Social en Uruguay. Revista Fronteras, (9), 29-45.

Alayón, N. (2007). Introducción para la 5a. edición. En N. Alayón, Historia del Trabajo Social en Argentina (pp. 13-23). Buenos Aires: Espacio.

Associação Brasileira de Ensino e Pesquisa em Serviço Social [ABEPSS]. (1996). Diretrizes Gerais para o Curso de Serviço Social: (Com base no Currículo Mínimo aprovado em Assembléia Geral Extraordinária de 8 de novembro de 1996). Recuperado de http://www.abepss.org.br/arquivos/textos/documento_201603311138166377210.pdf.

Battilana, N., y López, S. (2018). Incorporación de la Teoría Crítica en la formación profesional del Trabajo Social en la Universidad Nacional de Asunción de Paraguay. En V. Verbauwede, R. Zabinski, y L. Del Prado (Comps.), Formación en Trabajo Social: Miradas y reflexiones sobre el proceso de enseñanza (pp. 35-52). Paraná: Fundación La Hendija.

Bourdieu, P. (2008). El Campo Científico. En P. Bourdieu, Los usos sociales de la ciencia (pp. 11-57). Buenos Aires: Nueva Visión.

Bralich, J. (1993). La formación universitaria de Asistentes Sociales: más de seis décadas de historia. Montevideo, Uruguay: Universidad de la República.

Comité Latinoamericano y Caribeño de Organizaciones Profesionales de Trabajo Social/Servicio Social [(COLACATS]. (2012). Estatuto COLACATS. Recuperado de https://trabajosocial.or.cr/wp-

content/uploads/2020/10/1.1_ESTATUTO_COLACATS.pdf. 
Rivero-Rodríguez, Del Prado, Battilana-Amarilla y Coradini-Guilherme

Congreso de la Nación Argentina. (16 de Diciembre de 2014). Ley Federal del Trabajo Social [Ley 27.072 de 2014]. DO: 33.035.

Conselho Federal de Assistentes Sociais [CFAS]. (13 de marzo de 1993). Institui o Código de Ética Profissional dos Assistentes Sociais e dá outras providências [Resolución CFESS No. 273/1993].

Conselho Federal de Serviço Social [CFESS]. (2012). Código de ética do/a assistente social. Lei 8.662/93 de regulamentação da profissão. (10 ed.). Brasília: Fondos Murrado. Recuperado de http:/ / www.cfess.org.br/arquivos/CEP_CFESS-SITE.pdf.

Conselho Federal de Serviço Social [CFESS]. (2020). Perguntas frequentes. Recuperado de http:// www.cfess.org.br/visualizar/menu/local/perguntas-frequentes.

Coria, A., Badano, M., Achilli, E., Rosas-Pagaza, M., y Federación Argentina de Unidades Académicas de Trabajo Social [FAUATS]. (1999). Currículum e investigación en Trabajo Social. Buenos Aires: Espacio.

García, S. M. (1996). Bases para un análisis del origen del Trabajo Social en Paraguay. Asunción: Base. Investigaciones Sociales. Recuperado de http://www.baseis.org.py/wpcontent/uploads/2014/03/1395155043.pdf.

García, S. M. (2005). La Reconceptualización del Trabajo Social en Paraguay. En N. Alayón (Coord.), Trabajo Social Latinoamericano. A 40 años de la Reconceptualización (pp. 220-238). Buenos Aires: Espacio.

Garma, M. E., Acevedo, P., y Peralta, M. I. (2013). Precisiones conceptuales sobre lineamientos curriculares y condiciones institucionales. En P. Acevedo, y P. Fuentes, La formación académica en Trabajo Social en la República Argentina: Debates y desafíos (pp. 33-46). Córdoba: UNC-FAUATS.

Grassi, E. (1989). La mujer y la profesión de Asistente Social: el control de la vida cotidiana. Buenos Aires: Hvmanitas.

Iamamoto, M. V., \& Carvalho, R. de. (2003). Relações sociais e Serviço Social no Brasil: esboço de uma interpretação histórico-metodológica. São Paulo: Cortez.

Instituto Nacional e Estudos e Pesquisas Educacionais Anísio Teixeira [INEP]. (2019). Sinopse Estatística da Educação Superior 2018. Brasília: INEP. Recuperado de http:/ / inep.gov.br/sinopses-estatisticas-da-educacao-superior.

Jong, E. de, Ludi, M., y Badano, M. (2003). Formación académica en Trabajo Social: una apuesta política para repensar la profesión. Buenos Aires: Espacio.

Kuhn, T. (2006). La estructura de las Revoluciones Científicas. Buenos Aires: Fondo de Cultura Económica.

Martínez, S., y Agüero, J. (2014). La Ley Federal de Trabajo Social en Argentina: un caso de transformación profunda de un campo profesional. Revista Regional de Trabajo Social, 28(62), 84-93.

Melano, M. C., \& Deslauriers, J. P. (2012). El Trabajo Social latinoamericano: elementos de identidad. Buenos Aires: LUMEN-HVMANITAS.

Montaño, C. (2000). La naturaleza del Servicio Social. Un ensayo sobre su génesis, su especificidad y su reproducción. Sao Paulo: Cortez. 
Rivero-Rodríguez, Del Prado, Battilana-Amarilla y Coradini-Guilherme

Rivero, S. (2018). La continuidad de la ruptura. La formación de profesionales en Trabajo Social en Uruguay. En V. Verbauwede, R. Zabinski, y L. Del Prado, Formación en Trabajo Social. Miradas y reflexiones sobre el proceso de enseñanza (pp. 19-34). Paraná: Fundación La Hendija.

Rivero, S., y Prado, L. Del. (2018). Los “clásicos" en Trabajo Social. La formación en Uruguay. Sociedade em Debate, 24(3), 129-140.

Rivero, S., y Vecinday, L. (2005). Modalidades de Intervención en Trabajo Social. Material elaborado para el curso de Educación Permanente de Graduados: Definiciones estratégicas y modalidades de intervención en Trabajo Social. Recuperado de https://issuu.com/ale03/docs/_definiciones_estrat_gicas_y_modalidades_de_interv.

Rozas-Pagaza, M. (2017). Situación de la formación de grado y posgrado en Trabajo Social en el Cono Sur. En S. Cazzaniga, Entramados conceptuales en Trabajo Social. Categorías y problemáticas de la intervención profesional (pp. 90-106). Paraná: Editorial Fundación La Hendija.

Universidad Nacional de Asunción [UNA]. (1989). Propuesta Plan Curricular. Escuela de Trabajo Social [Resolución No. 2832-00-89].

Universidad Nacional de Asunción [UNA] (2015). Por la cual se aprueba el plan curricular de la carrera de Trabajo Social y el plan puente para la implementación del plan curricular de la carrera de Trabajo Social - 2016, del Instituto de Trabajo Social. Dependiente del Rectorado de la UNA [Resolución No. 0183-00-2015].

Universidad Nacional de Entre Ríos. Facultad de Trabajo Social. (2018). Programa “Fundamentos del Trabajo Social" [Resolución de Consejo Directivo No. 549/2018]. Recuperado de http://www.fts.uner.edu.ar/carreras/programas_ts_m/2018/1ro/1.2\%20LTS\%202 018\%20Fundamentos\%20de\%20TS.pdf.pdf.

Yazbek, M. C. (2009a). O significado sócio-histórico da profissão. En Conselho Federal de Serviço Social [CFESS] y Associação Brasileira de Ensino e Pesquisa em Serviço Social [ABEPSS] (Orgs.), Serviço Social: direitos sociais e competências profissionai (pp. 125-142). Brasília: CFESS/ABEPSS.

Yazbek, M. C. (2009b). Fundamentos Históricos e Teórico-Metodológicos em Serviço Social). En Conselho Federal de Serviço Social [CFESS] y Associação Brasileira de Ensino e Pesquisa em Serviço Social [ABEPSS] (Orgs.), Serviço Social: direitos sociais e competências profissionai (pp. 143-164). Brasília: CFESS/ ABEPSS. 


\section{OTROS ARTÍCULOS DE PROSPECTIVA No. 33}

\section{EDITORIAL}

El Paro Nacional en Colombia 2021: explosión social entre dinámicas estructurales y de coyuntura. Relevancia de la acción política y del diálogo en su desarrollo y transformación Adolfo Adrián Álvarez-Rodríguez

\section{ARTÍCULOS}

Organizaciones sociales de pobladores y prácticas de resistencia en contexto de pandemia COVID-19 en Chile

Patricia Castañeda-Meneses

Entre el aislamiento y las brechas digitales: sistematización de experiencia de acompañamiento socioemocional en personas mayores de Temuco, Chile, en tiempos de COVID-19

G. Bernarda Aedo-Neira

Percepción de apoyo social y calidad de vida: la visión de personas mayores chilenas en el contexto de pandemia durante el 2020

Claudia Elena Quiroga-Sanzana

Gabriela Rocío Parra-Monje

Camila Julia Moyano-Sepúlveda

Marco Alejandro Díaz-Bravo

A propósito del qué, cómo y para qué investigar en el campo de la justicia juvenil: La trastienda de un proceso de investigación en Rosario, Argentina Karina De Bella

Proyección social: reflexión sobre una experiencia virtual con adolescentes en época de pandemia en Sucre y Bolivar, Colombia

Claudia Yaneth Martínez-Mina
La intervención social mediante proyectos comunitarios en modalidad virtual en Mérida, México. Una perspectiva desde el Trabajo Social Claudia Isabel Tzec-Puch Amairani Aracelly Ceh-Alvarado Yanet Guadalupe González-Canul

Resignificación de los vínculos Sociales durante la pandemia de COVID-19: narrativas de personas que convergen en Conjuntos residenciales en Bogotá, Colombia

Andrea Marcela Reyes-García

Laura Daniela Molina-Ortiz

La paradoja de la familia doblemente recluida: clínica de una pandemia en Colombia

Norman Darío Moreno-Carmona

Juan José Cleves-Valencia

Ciencias sociales, mundo y pandemia: por un futuro posible

José Miguel Segura-Gutiérrez

Lina Paola Vásquez-Ávila

¿Qué es Trabajo Social? Los primeros vínculos de los estudiantes con la profesión: experiencias de Argentina, Brasil, Paraguay y Uruguay

Silvia Orieta Rivero-Rodríguez

Leonel Del Prado

Nidia Graciela Battilana-Amarilla

Rosilaine Coradini-Guilherme

Intervención profesional de trabajadores sociales de la universidad del Quindio, Colombia. Asuntos epistemológicos, metodológicos y ético-políticos

Ana María Gil-Ríos 
Reflexiones sobre la intervención social en lo rural: experiencias en el Magdalena Medio, Colombia

Claudia Milena Quijano-Mejía

Johana Linares-García

Construcción de rutas de atención integral a la convivencia escolar en Cali, Colombia: sistematización de la experiencia

Leidy Johana Prado-Montaño

Luz Helena López-Rodríguez

Alejandra Gutiérrez-Cárdenas

Experiencia del proceso de intervención familiar de los actores participantes en la Escuela para Familias del ICBF-Jamundí, Colombia Katherin Viviana Silva-Minotta

María José Forero-Izquierdo

Jimena del Pilar Jaramillo-Jaramillo

\section{RESEÑAS DE LIBROS}

Alerta global: políticas, movimientos sociales y futuros en disputa en tiempos de pandemia Jairo Crispín

El neoliberalismo como teología política. Habermas, Foucault, Dardot, Laval y la historia del capitalismo contemporáneo

José Francisco Desentis-Torres

La Reconceptualización del Trabajo Social en Colombia: Análisis histórico-crítico de las décadas de 1960-1970

Juan Pablo Sierra-Tapiro

ARTISTA INVITADO

Juan Camilo González

Prospectiva

\section{PROSPECTIVA}

Revista de Trabajo Social e Intervención Social

No. 33 • ene.-jun. 2022

e-ISSN: 2389-993X • Universidad del Valle 